\title{
Chip maker turns to self-assembly
}

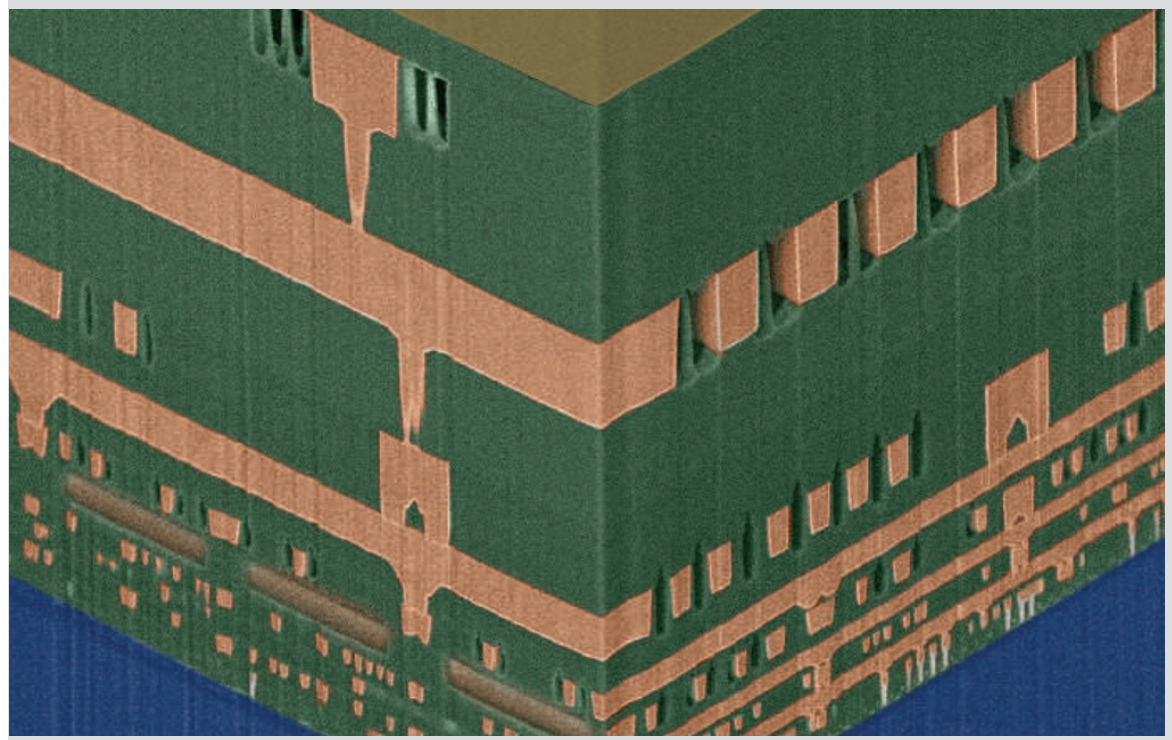

IBM has introduced a self-assembly process into chip manufacture - a first for the semiconductor industry. The company reports that the new chips consume 15\% less energy than conventional devices. Electrical signals can also travel 35\% faster in the new chips.

Dan Edelstein and co-workers exploit self-assembly to create air gaps — which are actually regions of vacuum - that act as insulators between the copper wires on the chip. A typical chip contains miles of wires, and as chips have become smaller, the wires inside them have become closer, making insulation more difficult. At first, semiconductor companies tried to solve this problem by introducing new materials with better insulating properties, but these materials tended to be fragile.

The IBM team has now developed a completely new solution that involves pouring a polymer onto a silicon wafer that has already been patterned with copper wires and a carbon silicate glass insulator, and then baking it to form a layer that contains trillions of holes, all measuring about 20 nanometres across. This array of holes then acts as a mask for a standard plasma etching process that drills vertical nanocolumns in the insulator. Subsequent steps dissolve the partitions between the nanocolumns to form continuous air gaps between the wires. Finally, the air is pumped out and another insulating layer is deposited to seal the vacuum gaps between the wires (www-03.ibm.com/press/us/en/ presskit/21463.wss).

The gaps can be seen beside the coppercoloured wires in the image, which measures about 15 micrometres across. The insulating layers are shown in green. The new process, which has been patented, has already been integrated into a manufacturing line at IBM's East Fishkill site in New York state, and is expected to be included in all the company's production lines by 2009 .

Peter Rodgers

\section{NANOMECHANICAL SYSTEMS Inside track weighs in with solution}

\author{
The performance of biosensors that rely on tiny vibrating cantilevers suffers when they are \\ operated in a liquid. The solution is to place the liquid inside the cantilever.
}

Javier Tamayo

is at the Institute of Microelectronics (IMM-CNM, CSIC), 28760 Madrid, Spain. e-mail: jtamayo@imm.cnm.csic.es

aboratories around the world are competing to measure the smallest possible masses with sensors based on tiny cantilevers that are fixed at one end and vibrate at the other. The concept is simple: when an object lands on the cantilever, its resonant frequency decreases by an amount that is proportional to the mass of the object. The sensitivity of the device is inversely proportional to the active mass of the cantilever, and as advances in fabrication techniques have allowed sensors to become smaller, the masses measured have evolved from picograms to zeptograms ${ }^{1-3}$. Improvements in transduction techniques which convert the vibration of the cantilever into a signal that can be measured - have also been central to this progress.

One of the driving forces in this field is the demand for biological sensors that are faster, more sensitive and capable of higher throughputs than current techniques ${ }^{4}$. Cantilever-based sensors offer many advantages: they can detect samples directly, whereas most existing techniques involve labelling the sample with fluorescent dyes, which is time-consuming and prone to false positives. Moreover, they can be reduced in size quite easily, which decreases the sample volumes. Finally, the ability to make devices with multiple cantilevers allows complex biochemical analysis. There is, however, one main disadvantage: biology tends to happen in aqueous solutions, whereas cantileverbased sensors perform best in a vacuum.

The key parameter is the quality or Q factor, which is significant in two ways: first 